\title{
SUBDOMINANT ULTRAMETRICS
}

\author{
JOSÉ M. BAYOD AND J. MARTÍNEZ-MAURICA
}

(Communicated by Dennis Burke)

\begin{abstract}
Given a metric space $(X, d)$ we study the existence of an ultrametric $\delta$ which is dominated by $d$. We give a number of examples and prove, among other results, that such an ultrametric exists if $(X, d)$ is locally compact and totally disconnected.
\end{abstract}

In some applications of ultrametric theory to real-world problems in physics and other sciences, it is of interest to know the precise relationship between a given metric $d$ on a certain finite set $X$ and the so-called subdominant ultrametric $\delta$, defined as the largest ultrametric on $X$ among those that are less than or equal to $d$ for every pair of points. The existence of such an ultrametric is trivial for any finite metric space, and some procedures are known for effectively computing the function $\delta$ in a combinatorial way; see [RAD] and [RTV] for these and other related questions.

Here we study the existence problem when the finiteness condition is dropped. We prove that the subdominant ultrametric exists and is equivalent to the given metric whenever the space is totally disconnected and locally compact and that it also exists for the metric completion of a space $X$ if it exists and is uniformly equivalent to $d$ over $X$. We also give a number of related examples. As a consequence of the first result, we prove very easily that a metrizable locally compact and totally disconnected space has covering dimension zero.

Throughout this paper, $(X, d)$ will be any metric space with more than one point. Observe that, given the collection $\left\{\delta_{i}: i \in I\right\}$ of ultrametrics over $X$ (i.e., metric functions fulfilling the strong triangular inequality $\forall x, y, z \in$ $\left.X, \delta_{i}(x, z) \leq \max \left\{\delta_{i}(x, y), \delta_{i}(y, z)\right\}\right)$ dominated by $d, \delta:=\sup \left\{\delta_{i}: i \in\right.$ $I\}$ is the largest ultrametric over $X$ dominated by $d$. In this case, we call $\delta$ the subdominant ultrametric of the space $(X, d)$. Obviously, existence of one ultrametric dominated by $d$ guarantees the existence of the subdominant ultrametric.

Received by the editors February 9, 1989 and, in revised form, July 7, 1989.

1980 Mathematics Subject Classification (1985 Revision). Primary 54E35; Secondary 54E45.

Key words and phrases. Ultrametric (space), subdominant ultrametric.

Research partially supported by grant 222 of Diputación Regional de Cantabria. 
On the other hand, defining for each pair $(x, y) \in X \times X$ the number $\delta_{X}(x, y)$ as the infimum of the set of positive numbers $\alpha$ such that

$$
\exists n \in \mathbf{N}, \exists\left\{a_{1}, \ldots, a_{n}\right\} \subset X, a_{1}=x, a_{n}=y,\left(\forall i<n, d\left(a_{i}, a_{i+1}\right)<\alpha\right),
$$

one obtains exactly the subdominant ultrametric of the space $(X, d)$ whenever it exists, i.e., whenever $\delta_{X}(x, y)>0$ for $x \neq y$.

Definition. We call the metric space $(X, d)$ preconnected when $\delta_{X}$ is identically zero and totally dispreconnected when $\delta_{X}$ is zero only on the diagonal, that is, when the subdominant ultrametric of $(X, d)$ exists.

This notion was introduced by G. Cantor (see [W, p. 312] for related historical remarks, including a complete reference to the original work of Cantor). We call it preconnectedness because it is the natural uniform counterpart of a characteristic property of connected spaces (cf. [W, Theorem 26.15]). It is clear that preconnectedness and total dispreconnectedness are uniform properties and can be defined on any uniform space. We will follow that direction eisewhere; here we will be concerned only about metric spaces.

The following example shows that the existence of a subdominant ultrametric is not a topological property:

Example 1. Define $X$ as the space of complex numbers $1,-1$, and all those $r e^{i \theta}$ such that for some $n \in \mathbf{N}, r=1-1 / n$ and $e^{i \theta}$ is an $n$th root of unity. Now let $Y$ be the space

$$
\{z \in X: \operatorname{Re} z<0\} \cup(1+\{z \in X: \operatorname{Re} z \geq 0\}) .
$$

The spaces $X$ and $Y$ are homeomorphic, but $Y$ is totally dispreconnected, unlike $X$, since $\delta_{X}(1,-1)=0$.

The space $\mathbf{Q}$ of rational numbers provides an example of a metric space with (covering) dimension zero but preconnected. Therefore, the existence of a compatible ultrametric does not guarantee the existence of the subdominant ultrametric.

As a consequence of Theorem 2, for locally compact metric spaces the existence of a compatible ultrametric is equivalent to the existence of the subdominant ultrametric. However, we next give an example of a complete metric space which is ultrametrizable but has no subdominant ultrametric.

Example 2. Consider the following sets of sequences of real numbers, where $n$ is a natural number, $n \geq 2$ :

$$
\begin{aligned}
& A_{n}=\left\{\left(x_{m}\right): x_{n}=k / n \text { with } k=0,1, \ldots, n \text { and } x_{m}=0 \text { if } m \neq n\right\} \\
& B_{n}=\left\{\left(x_{m}\right): x_{1}=1, x_{n}=k / n \text { with } k=0,1, \ldots, n \text { and } x_{m}=0 \text { if } 1 \neq m \neq n\right\} \\
& C_{n}=\left\{\left(x_{m}\right): x_{n}=1, x_{1}=k / n \text { with } k=0,1, \ldots, n \text { and } x_{m}=0 \text { if } 1 \neq m \neq n\right\}
\end{aligned}
$$


Let $X$ be the union of all these sets, and endow it with the sup distance

$$
d\left(\left(x_{m}\right),\left(y_{m}\right)\right)=\sup _{m}\left|x_{m}-y_{m}\right| .
$$

Then $\delta_{X}((0,0, \ldots),(1,0, \ldots))=0$.

As usual, we say that two metrics over the same set are topologically equivalent when they define the same topology and that they are uniformly equivalent when they define the same uniformity.

It is an open problem whether the existence of a subdominant ultrametric guarantees the existence of a topologically equivalent ultrametric. A simple modification of the last example gives a complete ultrametrizable metric space whose subdominant ultrametric exists but is not topologically equivalent:

Example 3. With the same notations as in Example 2, define

$$
B_{n}^{\prime}=\left\{\left(x_{m}\right): x_{1}=1, x_{n}=k / n \text { with }[n / 2] \leq k \leq n \text { and } x_{m}=0 \text { if } 1 \neq m \neq n\right\}
$$

and let $X^{\prime}$ be the union of all $A_{n} \cup B_{n}^{\prime} \cup C_{n}$ endowed with the sup distance as above. Then the sequence $\left(x^{n}\right)$ defined by

$$
x^{n}=(1,0, \ldots, 0,1 / 2,0, \ldots) \in B_{2 n}^{\prime}, n \in \mathbf{N},
$$

$\delta_{X^{\prime}}$-converges to $(0,0, \ldots)$, but is not $d$-Cauchy.

Theorem 1. Let $(X, d)$ be any metric space, and assume that there exists an ultrametric $\delta$ on $X$ uniformly equivalent to $d$. Then there also exists an ultrametric $\tilde{\delta}$ on the completion $(\tilde{X}, \tilde{d})$, uniformly equivalent to $\tilde{d}$. Moreover, if $\delta \leq d$, then $\tilde{\delta}$ can be chosen in such a way that $\tilde{\delta} \leq \tilde{d}$.

Therefore, on the space $\mathbf{Q}$ of rational numbers there is no ultrametric uniformly equivalent to the Euclidean metric.

Proof. Let us prove the first paragraph, from which the second paragraph follows.

Consider the completion of the uniform space associated with $(X, d)$ [or with $(X, \delta)$ ]. The two metrics $d$ and $\delta$ can be extended to the completion in a unique way to metrics $\tilde{d}$ and $\tilde{\delta}$ compatible with its uniformity. The statements in the first paragraph are now obvious.

Example 4. If $n \geq 2$, then $\mathbf{R}^{n}$ contains a discrete subspace $X$ that has a subdominant ultrametric topologically equivalent to the Euclidean metric such that there is no subdominant ultrametric on its completion $\tilde{X}$. Take $X$ as the space in Example 1 minus $\{1,-1\}$.

Therefore, the condition about uniform equivalence stated in Theorem 1 cannot be dropped in general.

However, as a consequence of Theorem 4, no such subspaces exist in $\mathbf{R}$.

Theorem 2. If $(X, d)$ is a locally compact and totally disconnected metric space, then there exists an ultrametric $\delta$ on $X$ dominated by $d$ and topologically equivalent to $d$. 
Proof. Let $x, y$ be two different points of $X$. Since $X$ is locally compact and totally disconnected, there is a compact open subset $K$ such that $x \in K$ and $y \notin K$. Then $\delta_{X}(x, y)$ cannot be less than the distance from $K$ to its complement; that is, it must be strictly positive. This proves that $(X, d)$ has a subdominant ultrametric.

Assume that $\left(x_{n}\right)$ is a sequence in $X$ and $x$ is a point in $X$ such that $d\left(x, x_{n}\right) \nrightarrow 0$. Then for some positive $\varepsilon$ and for infinitely many $n, d\left(x, x_{n}\right)>$ $\varepsilon$. Reasoning as above, we take a compact open set $K$ that contains $x$ and is contained in the $d$-ball of radius $\varepsilon$ around $x$; then for infinitely many $n$, $\delta_{X}\left(x, x_{n}\right)$ is greater than the $d$-distance from $K$ to its complement. Therefore $\delta_{X}$ and $d$ are topologically equivalent.

As a consequence of Theorem 2 , we prove very easily that every locally compact and totally disconnected metrizable space has covering dimension zero.

Example 5. Unlike the case when $(X, d)$ is compact, if $(X, d)$ is only locally compact, the conditions

(i) $\delta \leq d$ is an ultrametric

(ii) $\delta \leq d$ is an ultrametric topologically equivalent to $d$

are not equivalent. Take $X=\mathbf{N}, d=$ usual distance on $\mathbf{N}, d^{\prime}=$ the metric induced on $\mathbf{N}$ via the map $\mathbf{N} \rightarrow \mathbf{R}, 1 \mapsto 0, n \mapsto 1 / n$ if $n \geq 2$, and $\delta=$ an ultrametric dominated by $d^{\prime}$, which exists by Theorem 2 .

See, however, Theorem 4.

Example 6. Again unlike the case when $(X, d)$ is compact, if $(X, d)$ is locally compact and totally disconnected, its subdominant ultrametric may not be uniformly equivalent to $d$. Take

$$
X:=X^{\prime} \backslash\{(0,0, \ldots)\},
$$

where $X^{\prime}$ is the space of Example 3. Then the sequence $\left(x^{n}\right)$ defined by

$$
x^{n}=(1,0, \ldots, 0,1 / 2,0, \ldots) \in B_{2 n}^{\prime}, n \in \mathbf{N},
$$

is $\delta_{X^{\prime}}$-Cauchy but is not $d$-Cauchy.

Recall that a metric space $(X, d)$ is metrically convex if, given any two different points $x, y \in X$ and any $\lambda \in(0,1)$, there exists another point $z$ in $X$ such that

$$
d(x, z)=\lambda d(x, y) \quad \text { and } \quad d(y, z)=(1-\lambda) d(x, y) .
$$

Any metric space can be isometrically imbedded in a metrically convex space; consider the tight span introduced by A. Dress in [D]. The ultrametric equivalent has been studied by the authors in [BM].

Theorem 3. If $(X, d)$ is totally dispreconnected, then it is nowhere dense in any metrically convex space that contains it.

Proof. Assume that $Y$ is a metrically convex space that contains $X$, and $\operatorname{int}(\mathrm{Cl}(X)) \neq \varnothing$. Let $B\left(y_{0}, 3 r\right) \subset \mathrm{Cl}(X)$; since $Y$ is metrically convex, there exist two different points $a, b$ inside $B\left(y_{0}, r\right) \cap X$. 
Call $\lambda=d(a, b)$, let $\alpha$ be a positive real number not greater than $\lambda$, and let $n$ be the integer part of $\lambda / \alpha$; by metrical convexity of $Y$, there is a $y_{1}$ with

$$
d\left(a, y_{1}\right)=\alpha \quad \text { and } \quad d\left(y_{1}, b\right)=\lambda-\alpha .
$$

Inductively, we pick $y_{2}, \ldots, y_{n}$ with

$$
d\left(y_{k-1}, y_{k}\right)=\alpha \quad \text { and } \quad d\left(y_{k}, b\right)=\lambda-k \alpha \quad(2 \leq k \leq n) .
$$

All these $y_{k}$ belong to the ball $B\left(y_{0}, 3 r\right)$ because

$$
d\left(y_{k}, y_{0}\right) \leq k \alpha+d\left(y_{0}, a\right) \leq \lambda+r<3 r \quad(1 \leq k \leq n),
$$

and therefore they belong to $\mathrm{Cl}(X)$, so that we can choose $x_{k} \in X$ with $d\left(x_{k}, y_{k}\right)<\alpha \quad(1 \leq k \leq n)$. Then $\delta_{X}(a, b)=0$.

The converse of Theorem 3 is false, even for complete spaces of covering dimension zero, as the space of Example 2 shows: we need to prove only that if $Y$ is any metrically convex superspace of $X$, then the interior of $X$ in $Y$ is empty; consider in the space $Y$ any ball $B(x, r)$ with center in $X$, and let $x^{\prime}$ be any point in $X$ different from $x$. Since $Y$ is metrically convex, for any irrational number $t$ with $0<t<\min \left\{r, d\left(x, x^{\prime}\right)\right\}$, there is some $y \in Y$ such that

$$
d(x, y)=t \quad \text { and } \quad d\left(x^{\prime}, y\right)=d\left(x, x^{\prime}\right)-t .
$$

It is obvious that $y \notin X$, so that $X$ has no interior.

Nevertheless, for subspaces of $\mathbf{R}$ the following characterization can be proved:

Theorem 4. If $(X, d)$ is a metric subspace of $\mathbf{R}$, then the following properties are equivalent:

(i) there is an ultrametric that is dominated by $d$ and topologically equivalent to $d$;

(ii) $X$ is totally dispreconnected;

(iii) $\operatorname{int}(\mathrm{Cl}(X))=\varnothing$.

Proof. (i) $\Rightarrow$ (ii) is trivial, and (ii) $\Rightarrow$ (iii) follows from the previous theorem. To show that (iii) $\Rightarrow(\mathrm{i})$, let $\delta:=\delta_{X}$. Suppose there is a sequence $\left(x_{n}\right) \subset X$ and a point $x \in X$ such that $\delta\left(x, x_{n}\right)$ converges to zero but $d\left(x, x_{n}\right)$ does not tend to zero. Then there is a positive $\varepsilon$ and infinitely many $n$ 's satisfying $d\left(x, x_{n}\right)>\varepsilon$. Assume that there are infinitely many $n$ 's such that $x_{n}<x-\varepsilon$ (otherwise, for an infinity of $n$ 's, $x_{n}>x+\varepsilon$ ); then $[x-\varepsilon, x]$ is contained in $\mathrm{Cl}(X)$, against our hypothesis.

\section{ACKNOWLEDGMENT}

The authors wish to express their gratitude to the referee, who greatly simplified the proofs of Theorems 1 and 2. 


\section{REFERENCES}

[BM] José M. Bayod and J. Martinez-Maurica, Ultrametrically tight extensions, Proc. Amer. Math. Soc. 101 (1987), 571-576.

[D] Andreas W. M. Dress, Trees, tight extensions of metric spaces, and the cohomological dimension of certain groups: a note on combinatorial properties of metric spaces, Adv. in Math. 53 (1984), 321-402.

[RAD] R. Rammal, J. C. Angles d'Auriac, and B. Doucot, On the degree of ultrametricity, J. Physique Lett. 46 (1985), L-945-L-952.

[RTV] R. Rammal, G. Toulouse, and M. A. Virasoro, Ultrametricity for physicists, Rev. Modern Physics 58 (1986), 765-788.

[W] S. Willard, General topology, Addison-Wesley, Reading, MA, 1970.

Departamento de Matemáticas, Estadística y Computación, Universidad de CantaBRIA, 39071 SANTANDER, SPAIN 